\title{
INPUT SHAPING CONTROL TO REDUCE RESIDUAL VIBRATION OF A FLEXIBLE BEAM
}

\author{
QUOC CHI NGUYEN ${ }^{1}$, HA QUANG THINH NGO ${ }^{2}$ \\ ${ }^{1,2}$ Head of Control and Automation Laboratory, Hochiminh City University of Technology; \\ ${ }^{1}$ nqchi@hcmut.edu.vn; ${ }^{2}$ thinhpfiev2003@yahoo.com
}

\begin{abstract}
In this paper, three control algorithms based on input shaping method are developed to suppress the residual vibration of a flexible beam. The flexible beam is modeled as an under-damped system. Three input shapers, ZV, ZVD, and ZVDD, are used to control the flexible beam. The three control algorithms are implemented by using the Mechatrolink-III motion system. The experiments are performed to verify the effectiveness of the three control algorithms.
\end{abstract}

Keywords. Flexible beam, input shaping, industrial network, motion control, Mechatrolink-III, residual vibration.

\section{INTRODUCTION}

For industrial motion system, the vibration suppression is one of key techniques which researchers and engineers want to address the problem. Especially, with growth of accuracy manufacturing system, a need for the development of residual vibration technique has therefore arisen. One of efforts to suppress residual vibrations has been tried by modifying the motion profile. The proposed input motion design procedure in [1] was to define asymmetrical S-curve velocity profiles with fast acceleration and slow deceleration of AC servo motors which intermittently move the punching machine to desired positions with the reduced amplitude of residual vibrations. The authors in [2] developed an asymmetric S-curve profile method with jerk bounded to obtain high precision and reduce the residual vibration. Ha et al. [3] introduced a jerk ratio to scale down the jerks during the deceleration period. When the jerk ratio increases, the residual vibration decreases in motion system. Particularly, the high running speed can cause strong excitations in precision positioning machines. Thus, in [4], a low-vibration motion profile generation method to lessen systematic excitations was presented. The acceleration profile is designed by using a level-shifted sinusoidal waveform to have an S-shape in order to control its change rate. In $[5,6]$ the constraints between first natural frequency and deceleration time of motion profile was introduced. In these experiments, the acceleration, constant velocity and deceleration time intervals of trapezoidal velocity profile are selected by considering the lowest natural frequency at the stopping position. The root mean square (RMS) values are lowest if the inverse of the deceleration time equals to the first natural frequency. It is highest if the inverse of the deceleration time equals to the half of the first natural frequency.

Input shaping strategy is an attractive issue for reducing residual vibration in motion control system due to the robustness and effectiveness. Since Singhose et al. in [7] described a method for limiting vibration by adding constraints on the derivative of residual vibration magnitudes, various approaches to suppress the residual vibrations has been achieved such 
as hybrid input shaping [8], a three-impulse sequence input shaper [9] or lookup table control method [10]. However, Singhose's work performs the robustness to modeling errors and effectiveness to apply in the industrial motion system. In this method, engineer needs to determine the natural frequency and damping ratio in order to build an input shaping controller. Recently, researchers in [11] studied the effect of natural frequency error to residual vibration in three input shaping controller. ZVD and ZVDD shaper are more robust than ZV shaper if the error in natural frequency of flexible beam exists.

Mechatrolink-III protocol is a real-time protocol that is based on Ethernet technology and was developed by Yaskawa [12]. Mechatrolink-III is chosen to investigate because of their advantages such as fast communication, high reliability and rapid design. The performance of Mechatrolink-III was analyzed in [13] and slave station was designed in [14]. The protocol guarantees the short cycle time which is considered as a typical factor in motion control fields.

In this paper, the input shaper ZV, ZVD, and ZVDD are applied to suppress the residual vibration of a flexible beam when the beam moves to the desired position. The control algorithm is implemented using Mechatrolink-III. The experiments are carried out to verify the effectiveness of the control algorithm.

This paper is organized as follows. In Section 2, the model formulation of flexible beam is presented. Section 3 proposes the implementation of input shaper as an engineer solution. In Section 4, the hardware development and software development of Mechatrolink-III motion controller are shown. In Section 5, the experimental verifications and results are carried out. Finally, this paper ends with conclusions in Section 6.

\section{FLEXIBLE BEAM MODEL}

Consider a mass mounted on the end of flexible beam as Fig.1 (a). Both of the beam and the mass of the tip end are made from aluminum alloy. The material parameters of the beam are as follows. The beam has length $L$ and mass per unit length $\rho$, as shown in Fig.1 (a). The modulus of elasticity is $E$, and the internal bending moment of the beam is $I$.

Assuming that the end-mass is much greater than the mass of the beam. Define $m_{t}$ as the total mass at the tip end of the beam. It should be noted that $m_{t}$ can be computed as follows.

$$
m_{t}=m_{\text {beam }}+m,
$$

where $m_{\text {beam }}$ is the effective mass of the beam, and $m$ is the mass of the tip end. Define the effective mass of the beam $m_{\text {beam }}$ as

$$
m_{\text {beam }}=\rho \int_{0}^{L}\left[\frac{y(x)}{y_{\max }(L)}\right]^{2} d x .
$$

Using the first mode, the shape function of the beam (without the mass of the tip end $m$ ) can be obtained as follows. 


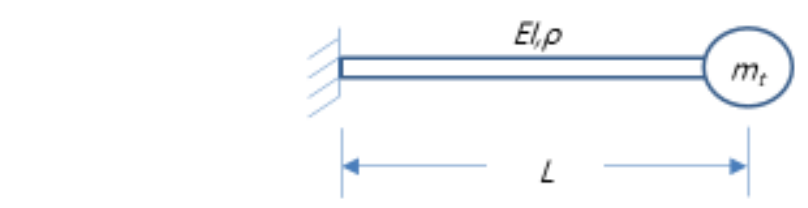

(a)

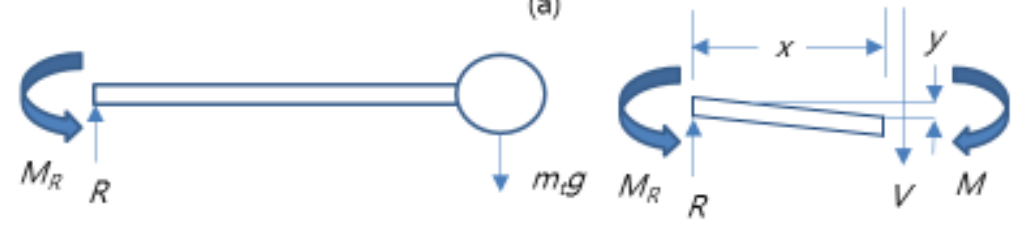

(b)

(c)

Figure 1. (a) Cantilever beam model; (b) free-body diagram; (c) a section of beam

$$
y(x)=\frac{\rho L g}{E I}\left(-\frac{x^{3}}{6}+\frac{L x^{2}}{2}\right),
$$

where $y(x)$ is the deflection of the static beam.

Substituting Eq. (3) to Eq. (2) yields.

$$
m_{\text {beam }}=0.23357 \rho L .
$$

The free-body diagram of beam is described in Fig.1 (b). Let $R$ be the reaction force, and $M_{R}$ be the reaction bending moment. Applying Newton's law for static equilibrium point, the following equations can be obtained.

$$
\begin{gathered}
R-m_{t} g=0, \\
M_{R}-m_{t} g L=0 .
\end{gathered}
$$

In Fig.1 (c), at the left boundary of the flexible beam, the sum of bending moments $M$ at the right side can be as follows.

$$
M=M_{R}-R x .
$$

Based on the differential equations for the elastic curve of a beam [13, Eq. (9.12), p. 297], the relation of the bending moment $M$ and the deflection $y(x)$ of the flexible beam can be obtained as follows.

$$
M=-E I \frac{d^{2} y(x)}{d x^{2}} .
$$

It should be noted that the curvature is negative. Substituting Eq. (8) into Eq. (7), results in the following equation at the right boundary. 


$$
\frac{d^{2} y(L)}{d x^{2}}=-\frac{m g}{E I}(L-x) .
$$

Integrating Eq. (9) twice with the zero boundary conditions

$$
\begin{gathered}
\frac{d y(L, 0)}{d x}=0, \\
y(L, 0),
\end{gathered}
$$

The following equation that describes the deflection of the tip of the beam (at right boundary) is obtained.

$$
y(L)=\left[\frac{m_{t} g L^{3}}{3 E I}\right] .
$$

In this paper, the flexible beam with total mass $m_{t}$ at the end is assumed to be a linear spring with the stiffness.

$$
k=\frac{3 E I}{L^{3}} .
$$

The natural frequency $\omega_{n}$ of the cantilever beam at the tip end can be computed as.

$$
\omega_{n}=\left(\frac{1}{2 \pi}\right) \sqrt{\frac{3 E I}{m_{t} L^{3}}} .
$$

Theoretically, the damping ratio $\zeta$ of an under-damped system in the time domain can be identified by using logarithmic decrement method in Fig. 2. The amount of logarithmic decrement $\delta$ is the natural logarithm of the ratio between the two amplitudes consecutive peaks $x_{i}$ and $x_{i+1}$.

$$
\delta=\frac{1}{n} \ln \frac{x_{i}}{x_{i+n}} .
$$

Then, the damping ratio is defined as follows.

$$
\zeta=\frac{\delta}{\sqrt{(2 \pi)^{2}+\delta^{2}}} .
$$

\section{INPUT SHAPER DESIGN}

In this paper, the control objective is to suppress the residual vibration while the beam is driven to the desired angle. It is assumed that the flexible beam system can be considered as an under-damped second order system, which can be expressed as the transfer function $G(s)$.

$$
G(s)=\frac{Y(s)}{U(s)}=\frac{\omega_{n}^{2}}{s^{2}+2 \zeta \omega_{n} s+\omega_{n}^{2}} .
$$

In the time domain, the impulse response of the system is given as 


$$
y(t)=\frac{A \omega_{n}}{\sqrt{1-\zeta^{2}}} e^{-\zeta \omega_{n}\left(t-t_{0}\right)} \sin \left(\omega_{n} \sqrt{1-\zeta^{2}}\left(t-t_{0}\right)\right),
$$

where $A$ and $t_{0}$ are the amplitude and the time of impulse, respectively. Basic technical point of input shaping is described in Fig. 3. When first impulse is applied, it results in vibration to a system (solid line). Then, if we continue to apply second impulse with appropriate amplitude and time location, this

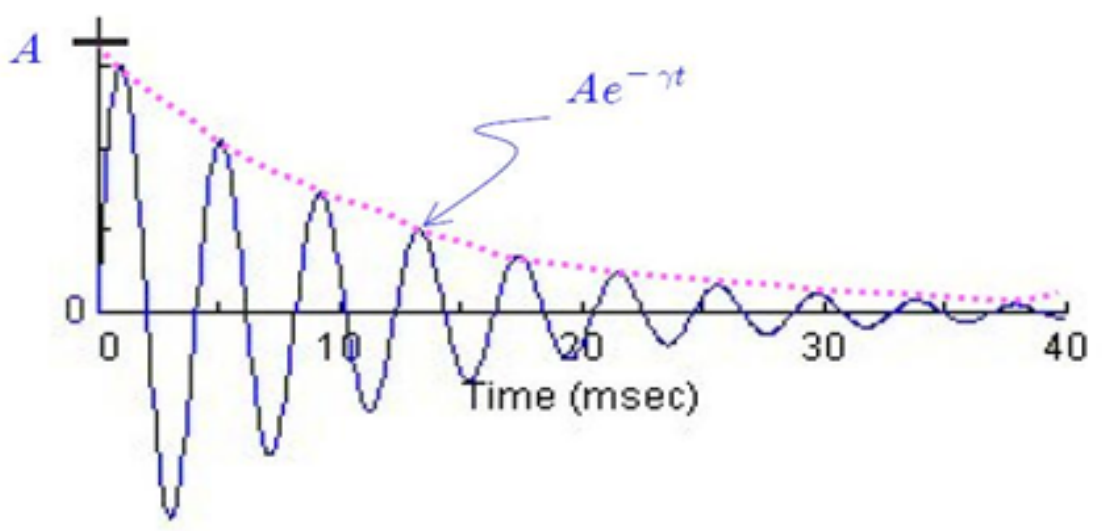

Figure 2. Illustration of logarithmic decrement method to calculate the damping ratio

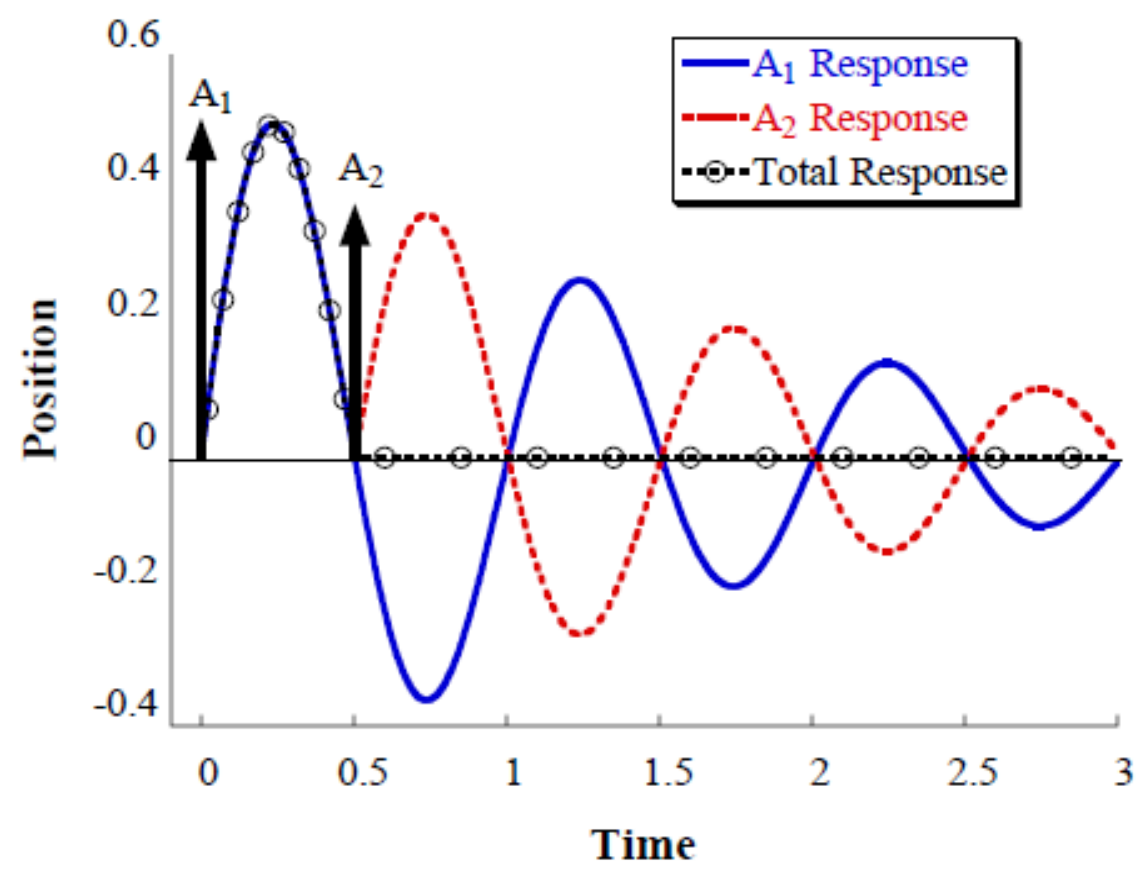

Figure 3. Basic concept of input shaping [7] 
impulse generates vibration (dash line), which can suppress the vibration caused by first impulse. This effect results in zero vibration of the system. A sequence of impulses that caused no vibration when applying to system is called input shaper.

The percentage residual vibration between the single unity-magnitude sequences is given as $[7]$

$$
V\left(\omega_{n}, \xi\right)=e^{-\xi \omega_{n} t_{n}} \sqrt{C\left(\omega_{n}, \xi\right)^{2}+S\left(\omega_{n}, \xi\right)^{2}}
$$

where

$$
\begin{aligned}
& C\left(\omega_{n}, \xi\right)=\sum_{i=1}^{n} A_{i} e^{\xi \omega_{n} t_{i}} \cos \left(\omega_{d} t_{i}\right), \\
& C\left(\omega_{n}, \xi\right)=\sum_{i=1}^{n} A_{i} e^{\xi \omega_{n} t_{i}} \cos \left(\omega_{d} t_{i}\right) .
\end{aligned}
$$

In the Eqs. (20) and (21) $A_{i}$ and $t_{i}$ is the amplitude and time location of the $i$-th impulse, $n$ is the number of impulses in the impulse sequence, $\omega_{n}$ is natural frequency, $\zeta$ is damping ratio, and $\omega_{d}=\omega_{n} \sqrt{1-\zeta^{2}}$ is defined as damped natural frequency.

$Z V$ is the simplest and earliest input shaper, it can be obtained by solving following constraints [7]:

$$
\begin{aligned}
& \text { 1) } V(\omega, \zeta)=0 \\
& \text { 2) } \sum A_{i}=1 \\
& \text { 3) } A_{i}>0 \\
& \text { 4) } t_{1}=0 \\
& \text { 5) } \min \left(t_{n}\right) .
\end{aligned}
$$

$Z V$ input shaper is given as the following matrix.

$$
\left[\begin{array}{c}
A_{j} \\
t_{j}
\end{array}\right]=\left[\begin{array}{cc}
\frac{1}{1+K} & \frac{K}{1+K} \\
0 & 0.5 T_{d}
\end{array}\right]
$$

where $T_{d}=2 \pi / \omega_{d}$ is the damped period of vibration, and $K=\exp \left(-\xi \pi / \sqrt{1-\xi^{2}}\right)$.

In this paper, the robust input shaper $Z V D$ [15] is also used to improve the control performance of the flexible beam system.

$$
\left[\begin{array}{l}
A_{j} \\
t_{j}
\end{array}\right]=\left[\begin{array}{ccc}
\frac{1}{J_{Z V D}} & \frac{2 K}{J_{Z V D}} & \frac{K^{2}}{J_{Z V D}} \\
0 & 0.5 T_{d} & T_{d}
\end{array}\right]
$$

where

$$
J_{Z V D}=1+2 K+K^{2} .
$$

To achieve more robustness than ZVD, the input shaper ZVDD [15] is introduced as follows.

$$
\left[\begin{array}{l}
A_{j} \\
t_{j}
\end{array}\right]=\left[\begin{array}{cccc}
\frac{1}{J} & \frac{3 K}{J} & \frac{3 K^{2}}{J} & \frac{K^{3}}{J} \\
0 & 0.5 T_{d} & T_{d} & 1.5 T_{d}
\end{array}\right]
$$


where

$$
J=K^{3}+3 K^{2}+3 K+1 .
$$

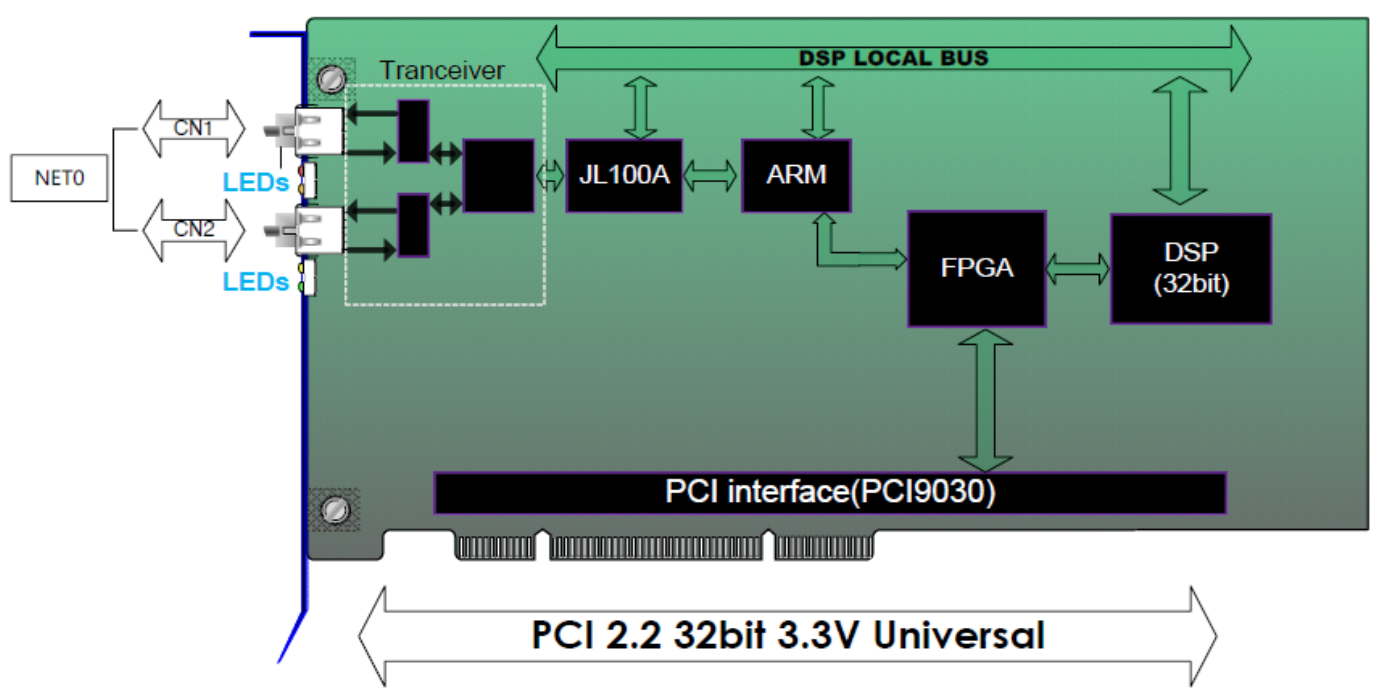

Figure 4. Block diagram of hardware design

\section{CONTROL IMPLEMENTATION}

In this paper, the controller of the flexible beam system is built by using the MechatronlinkIII network components, which includes one $\mathrm{C} 1$ master and various slaves which can be servo drive, stepping motor and I/O module. They can be connected in cascade, star or mix topology if using hub. In the network, C1 master receives command profiles from slaves and determines the kind of devices. After master sends commands, slaves receive and execute commands, and later slaves reply their monitoring information. The speed of network to transmit data is at $100 \mathrm{Mbps}$. The Mechatrolink-III protocol supports both synchronous and asynchronous communication modes. In synchronous mode, master station sends the command data at any required timing and the slave station responds to the sent command data. Asynchronous communication can be used in a system where synchronous operation is not needed, i.e., collecting necessary information for synchronous communication from slaves.

\subsection{Hardware development}

In this section, the design of Mechatrolink-III master device is introduced. Figure 4 presents the block diagram of hardware design. The controller communicates with host PC by PCI interface. Then, FPGA chipset is used for timing synchronization of controller. Based on the powerful and fast computation, motion generator and other calculations of signals are embedded into DSP. In this diagram, ARM CPU plays an important role. It handles data between internal controller and Mechatrolink-III network. Finally, ASIC JL-100A chip control Mechatrolink-III sending/receiving frame inside network. In Table 1, specifications of Mechatrolink-III controller are listed. 


\subsection{Software development}

In Windows platform, the static library 32-bit is programmed to input data from end-user. The essential parameters to apply input shaping are shown.

$$
\text { AxmAdvISTSetParameter(lAxis,dFreq,dDampingRatio,dImpulseCount) }
$$

where lAxis is the number of axis to control; dFreq and dDampingRatio are natural frequency and damping ratio of motion system. dImpulseCount displays the number of impulse to convolve.

$$
\begin{aligned}
& \text { dImpulseCount }=2: \text { ZV control scheme } \\
& \text { dImpulseCount }=3: \text { ZVD control scheme } \\
& \text { dImpulseCount }=4: \text { ZVDD control scheme }
\end{aligned}
$$

\section{AxmAdvISTEnable(1Axis,dEnable)}

The value TRUE of dEnable is to activate the input shaping technology, otherwise normal motion is execute. Once, natural frequency and damping ratio are input, amplitude and time cycle of impulse are known. A number of impulses depend on input shaping modes. When input shaping control is activated, a reference data from profile generator is driven into ZV, ZVD or ZVDD shaper scheme.

In low level, in Fig. 5, a loop which is responsible for the real-time control of data exchange is defined. The firmware includes the main program and interrupts service routine. In the first stage, PLL clock, timer, system control register, interrupt service routine and motion parameters are predetermined. Later, ASIC JL-100A is initialized in three times. If error still occurs, LED displays to notify. Otherwise, infinite loop that switches between asynchronous and synchronous mode is used to update data. As shown in Fig. 6, the input shaping strategies are implemented in ISR. After confirming the error system is in a range, motion profile is generated. Value of time is computed and compared with sampling time in system to determine the period of impulse. Then, a mathematical operation is convolved between two signals as an output of input shaper. The result is transmitted to slave station in Mechatrolink-III network in order to execute command profile.

Table 1. Specifications of Mechatrolink-III controller

\begin{tabular}{|l|l|}
\hline Form Factor & PCI $(32$ bit/33 MHz $)$ \\
\hline Protocol Communication & Mechatrolink-III \\
\hline Speed & $100 \mathrm{Mbps}$ \\
\hline Transmission Cycle & 250 us \\
\hline Data Frame & 48 byte \\
\hline Support Communication & Cyclic, Event-driven \\
\hline Mechatrolink-III Chipset & JL100A \\
\hline
\end{tabular}

The input shaping for S-curve motion profile is illustrated in Fig. 7. In fact, the conventional motion controller generating S-curve trajectory to drive AC Servo motor yield vibrations of the beam. In the case of the input shaping applied, the convolution product between S-curve profile and two-pulse command yield the better results, i.e., the dynamic 

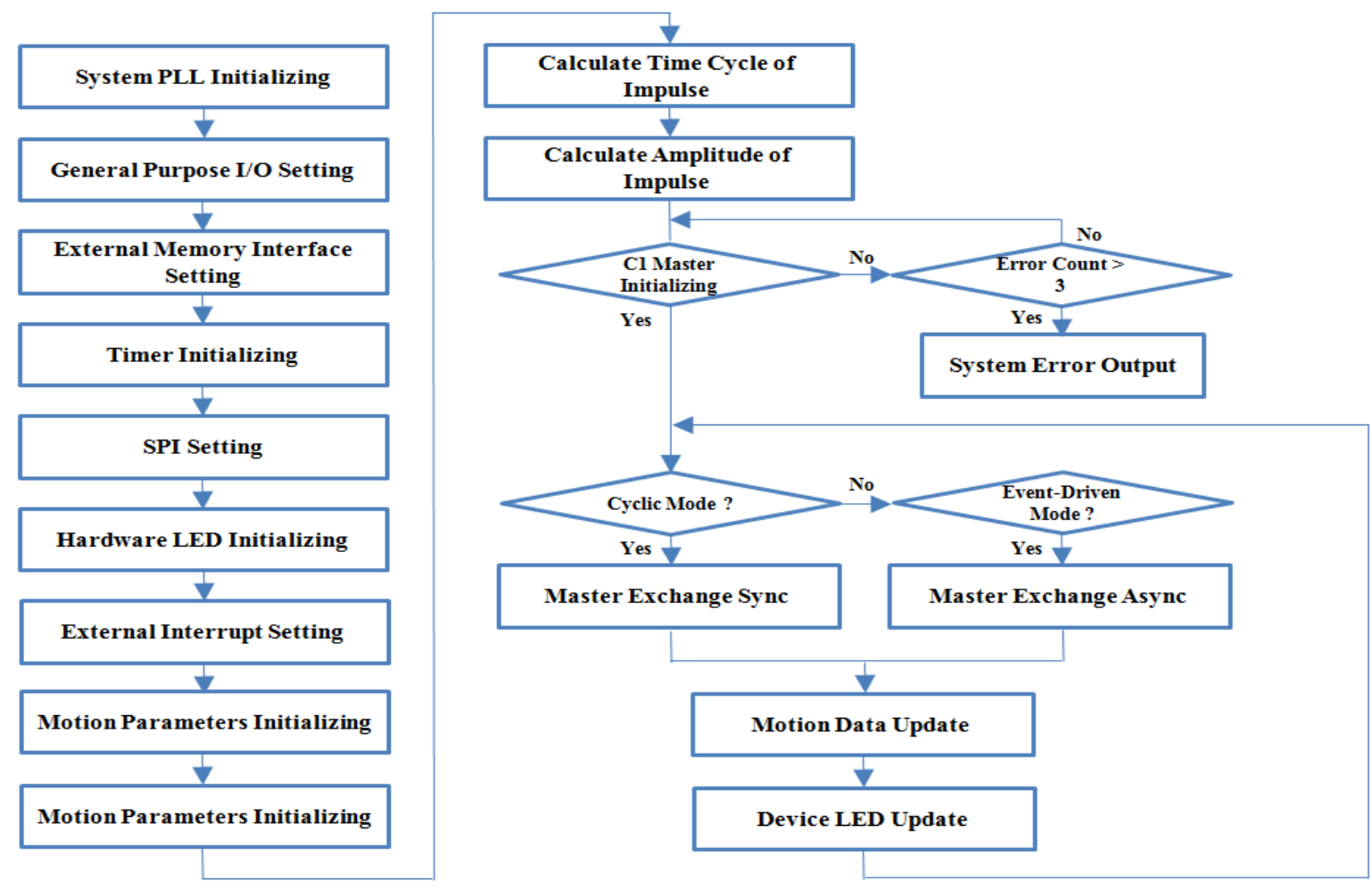

Figure 5. Flowchart of main program

response is smoother, and the residual vibration is suppressed when the motion is completed. The difference between the conventional and the proposed methods is the suppression of the vibration at the end of the motion. In the case of the conventional method, the vibration suppression is based on the viscous damping of the system. This can be considered as a passive vibration suppression method. In the case of the proposed control method, the vibration eliminated at the time the motion is completed. Therefore, the proposed control method may yields two advantages: (i) The vibration is suppressed quickly; (ii) The time for vibration suppression can be predicted.

\section{EXPERIMENTAL RESULTS}

Experiments were conducted with proposed Mechatrolink-III controller and slave servo drive as shown in Fig. 8 and its corresponding parameters can be seen in Table 2. An aluminum thin beam is used as flexible beam. Two bolts are hanged in each side of beam as mass or load. Then the beam is directly mounted with motor shaft which is vertically suspended on frame.

In order to identify exact natural frequency of beam, a laser sensor is used to measure the vibration the flexible beam. The flexible beam rotates around vertical direction and then, laser sensor detects residual vibration of beam. In Figs. 9 and 10, spectrum frequency and system vibration that measured by laser sensor are displayed. The measured natural frequency is $33.3 \mathrm{~Hz}$. Hence, the calculated damping ratio based on logarithmic decrement 


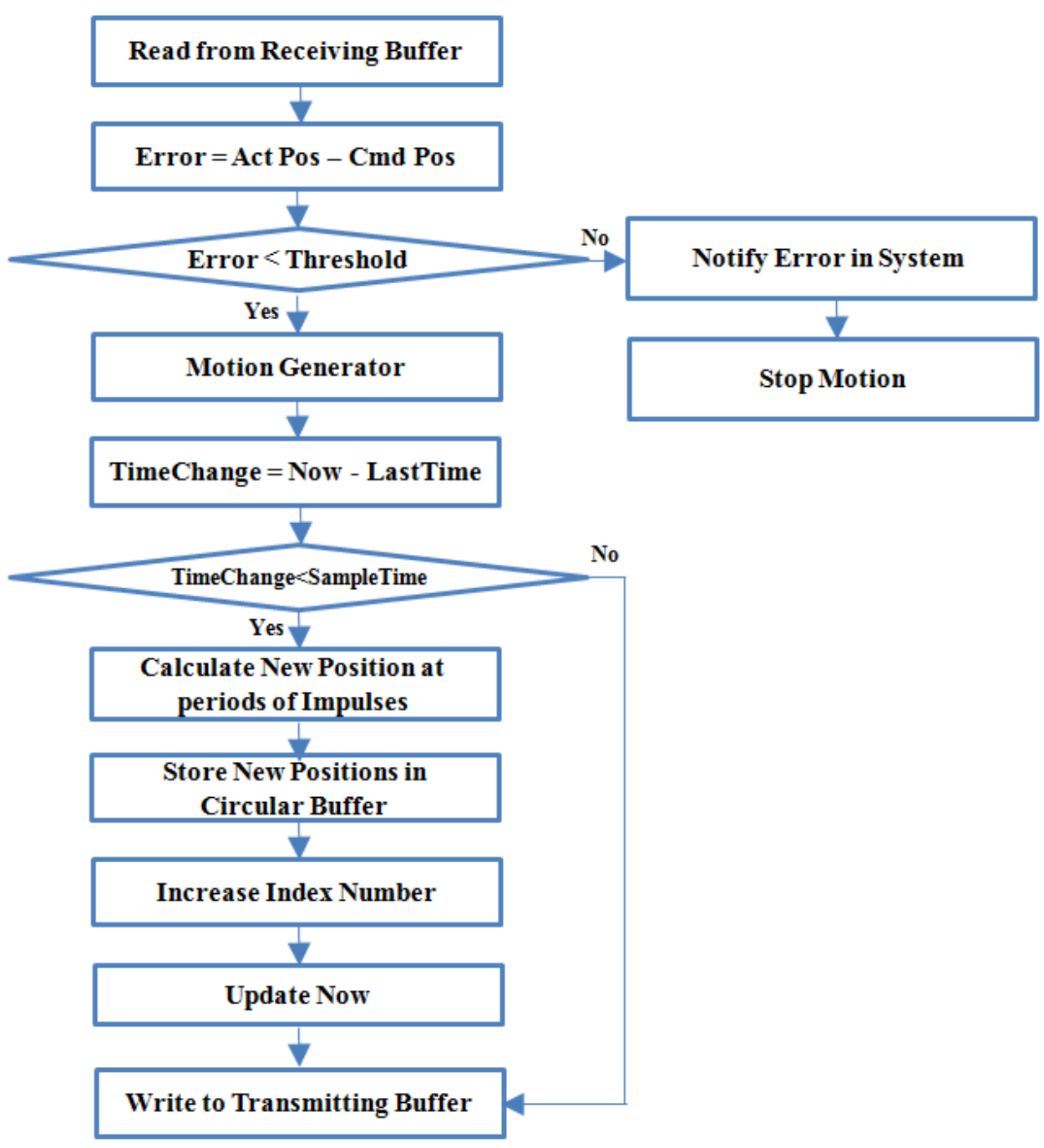

Figure 6. Flowchart of interrupt service routine

method is 0.008. These parameters are used in all tests. However, depending on each kind of input shapers, impulse counts are selected as 2,3 or 4

Table 2. Parameters of experimental motion system

\begin{tabular}{|l|l|}
\hline Young's modulus of beam & $70 \mathrm{GPa}$ \\
\hline Density & $2.7 \mathrm{~g} / \mathrm{cm}^{3}$ \\
\hline Length of beam & $85 \mathrm{~mm}$ \\
\hline Width of beam & $15 \mathrm{~mm}$ \\
\hline Height of beam & $1.8 \mathrm{~mm}$ \\
\hline Mass of two bolts & $1.2 \mathrm{~g}$ \\
\hline
\end{tabular}

The experimental results of the case of ZV, ZVD and ZVDD shapers and of the conven- 


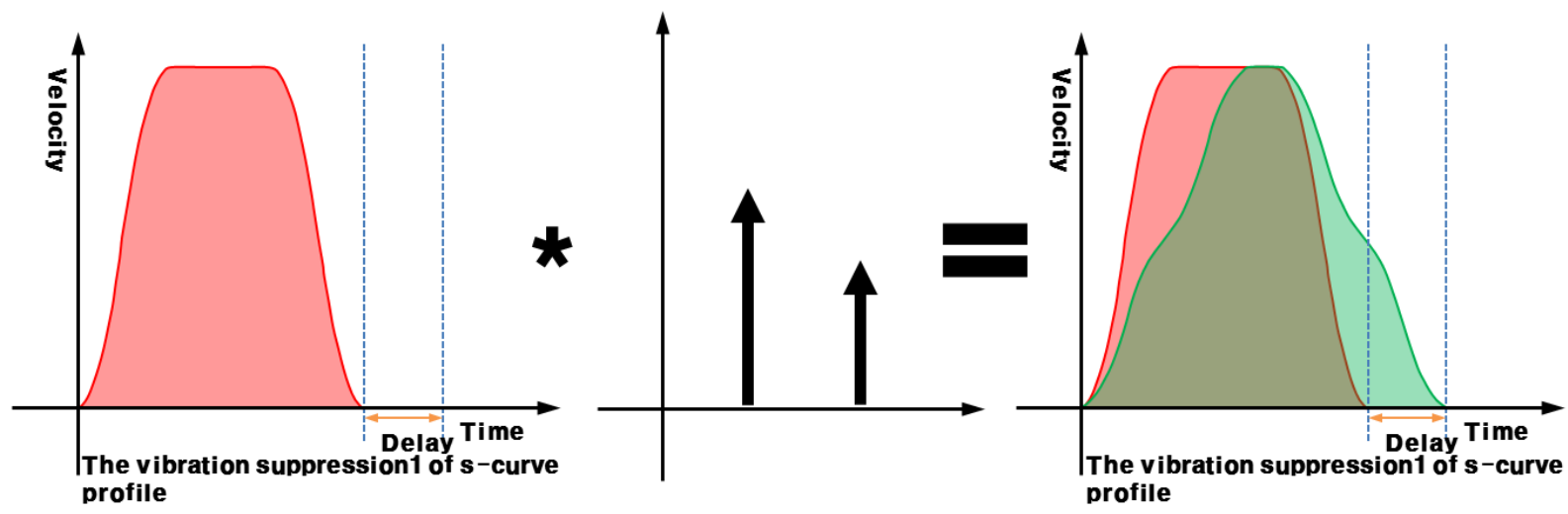

Figure \%. Input shaping for S-curve profile

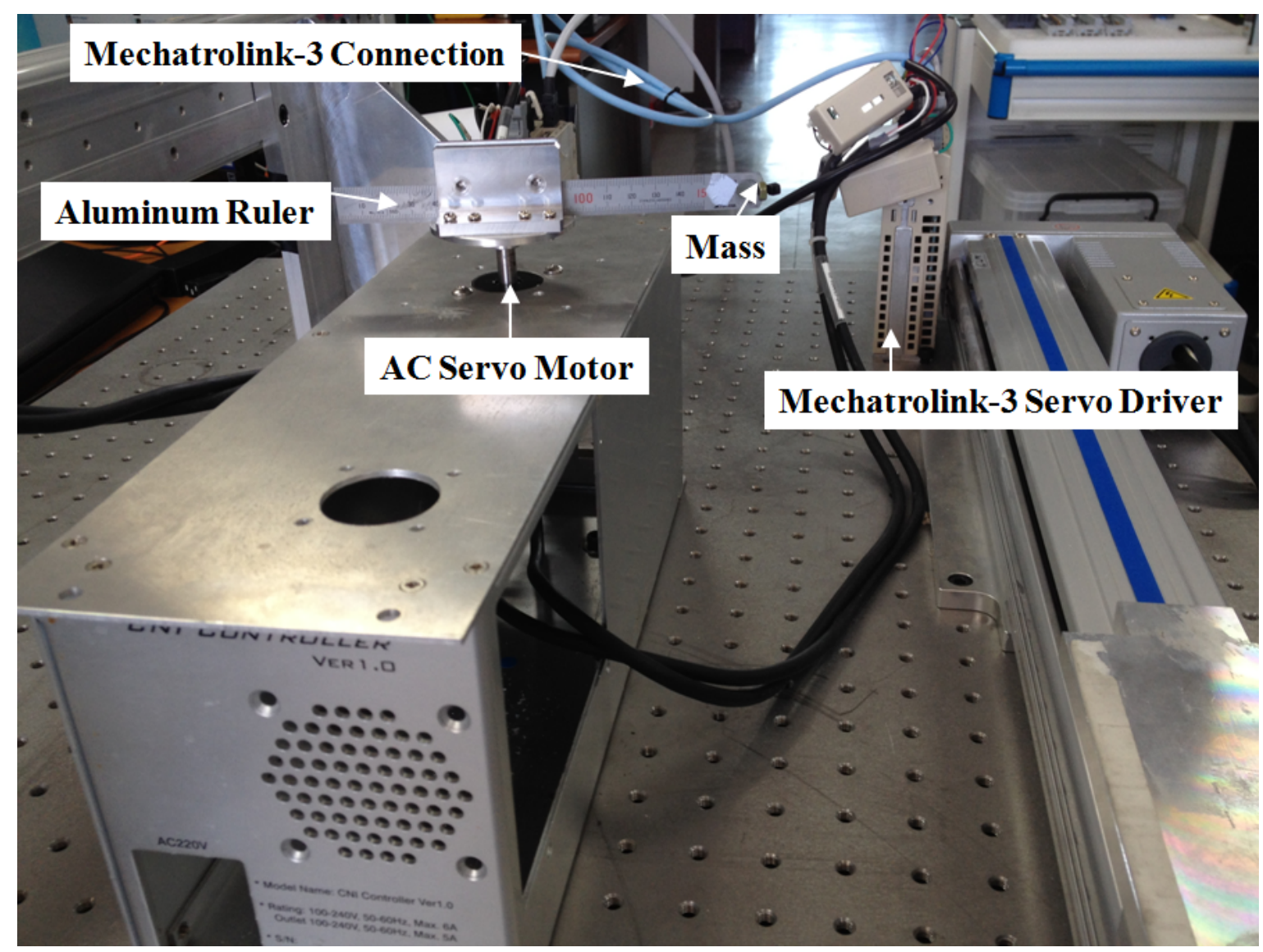

Figure 8. Experiment system is set-up

tional method are described in Figs. 11, 12, 13 and 14 correspondingly. To illustrate visually, speed and position of servo motor are plotted on left and right side of vertical axis. Their units are revolutions per minute (rpm) and the number of rotations (rev) around motor shaft. In these experiments, angle of the beam is obtained by using the 17-bit incremental 


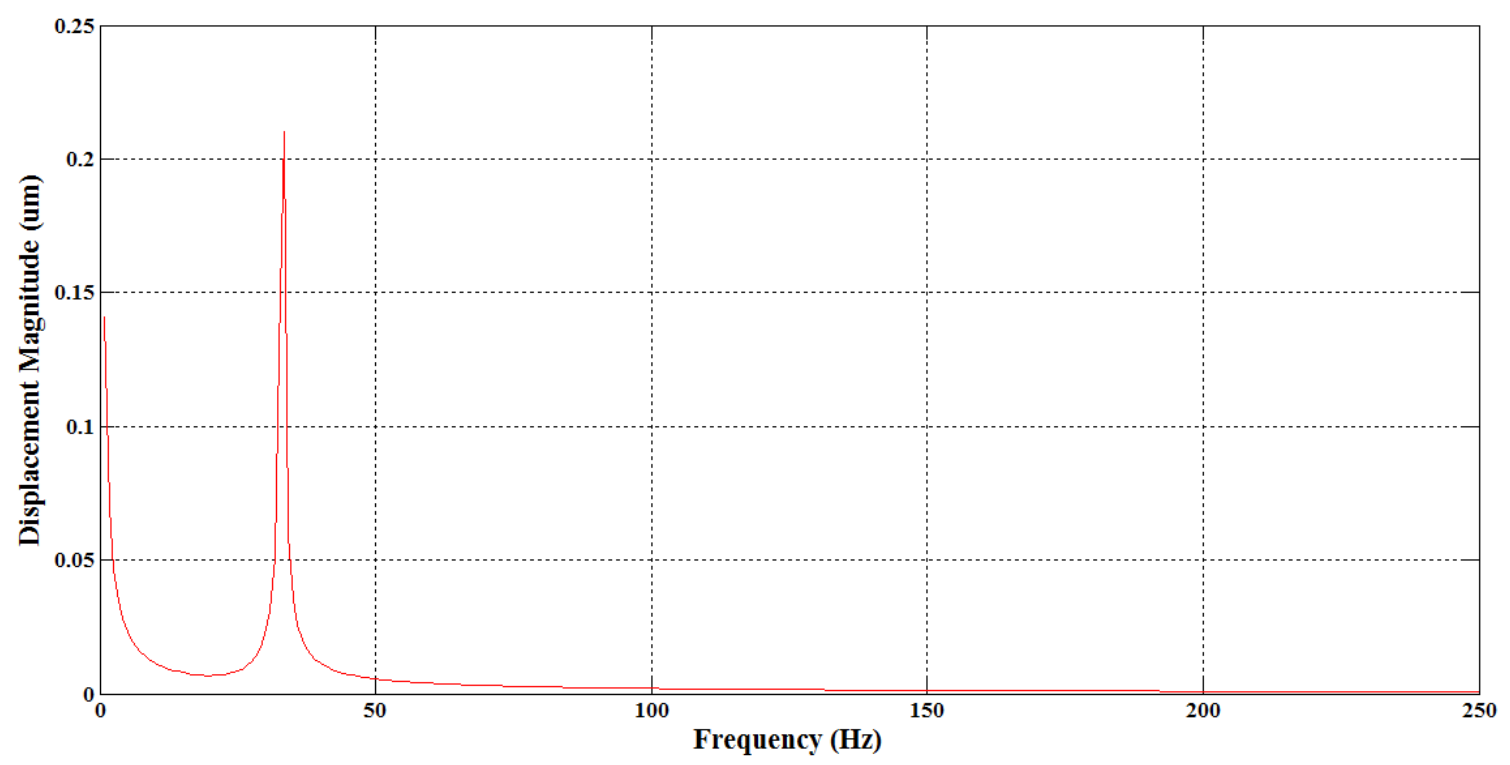

Figure 9. Spectrum frequency measured by laser sensor

encoder in the servo motor. Based on a motion planning trajectory, controller generates the reference (command) speed to drive AC servo motor and obtains the actual speed. Fig. 11 shows the results in the case of the conventional method applied. There is the oscillation in the actual speed, especially in period of constant velocity and deceleration. When the $\mathrm{ZV}$ shaper is applied, the oscillation appears in the speed signal is decreased in comparison to the case of the conventional method, as shown in Fig. 12. The ZVD and ZVDD shapers yield the better results than ZV, as shown in the Figs. 13 and 14, where the oscillations are suppressed completely. Fig. 15 shows experimental data of torque control in the case of ZV, ZVD and ZVDD input shapers and of the conventional method. It is obvious that there is the torque of the conventional method oscillates with a big amplitude There are still small residual vibrations with the ZV and ZVD shapers, but residual vibrations are zero in the case of the ZVDD shaper applied The experiments show that input shapers yield good performance in Mechatrolink-III motion control system

Table 3. Comparison of RMS tracking errors and reduction ratios in test cases

\begin{tabular}{|l|l|l|}
\hline Control Scheme & RMS & Reduction \% \\
\hline Without Input Shaper & $1.4721 \mathrm{e}-3$ & - \\
\hline With ZV Input Shaper & $1.0572 \mathrm{e}-3$ & 28.18 \\
\hline With ZVD Input Shaper & $0.7233 \mathrm{e}-3$ & 50.86 \\
\hline With ZVDD Input Shaper & $0.2615 \mathrm{e}-3$ & 82.23 \\
\hline
\end{tabular}

The comparison of the control performance of the three proposed control methods, which has been illustrated, is shown in Table 3. It should be noted that the tracking error is an important factor to evaluate motion control systems. In the case of the conventional control, the experimental result shows large RMS tracking error. The input shaper ZV, ZVD, and ZVDD, yield the reduction of the RMS tracking error, where ZVDD generates the best 


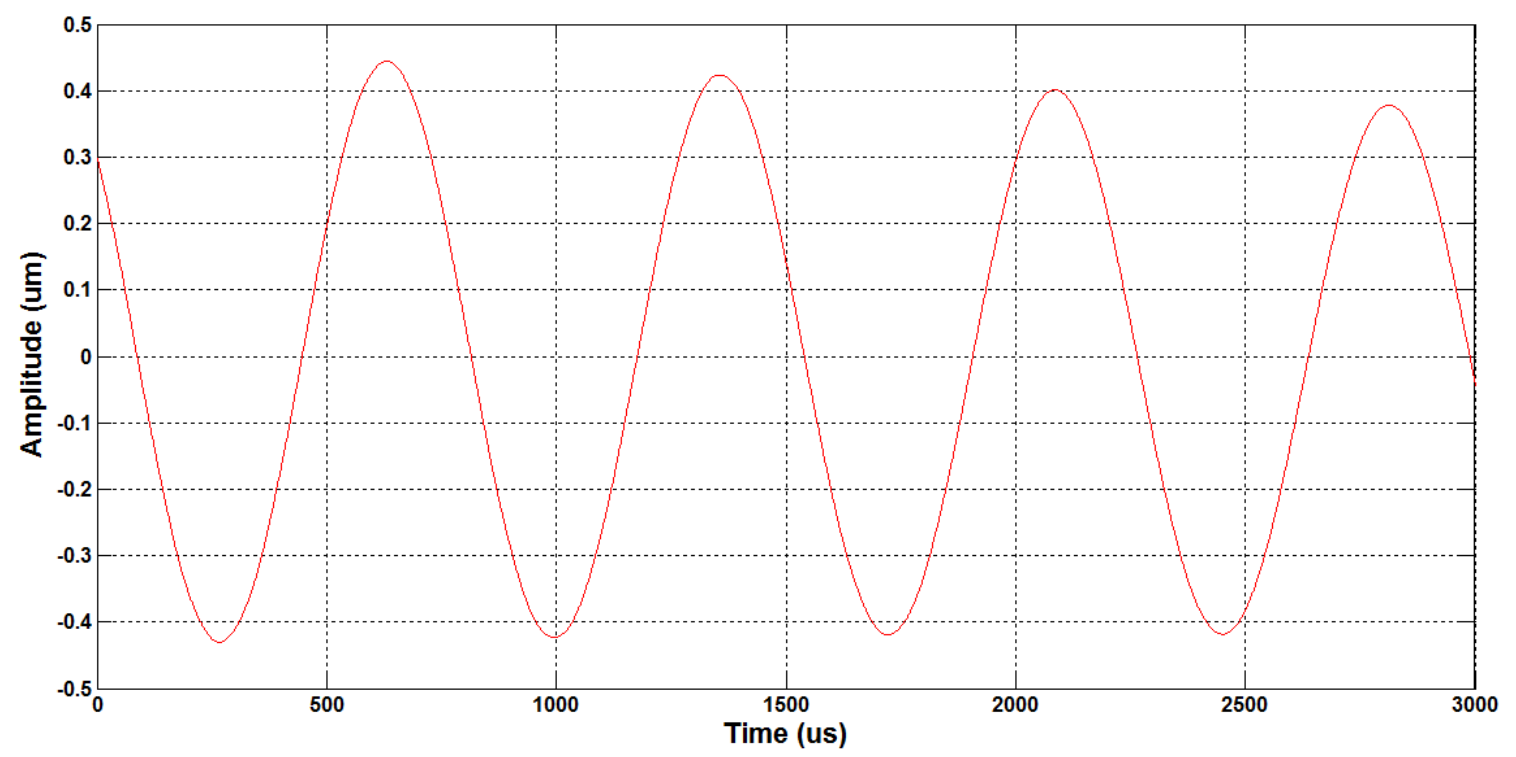

Figure 10. System vibration measured by laser sensor

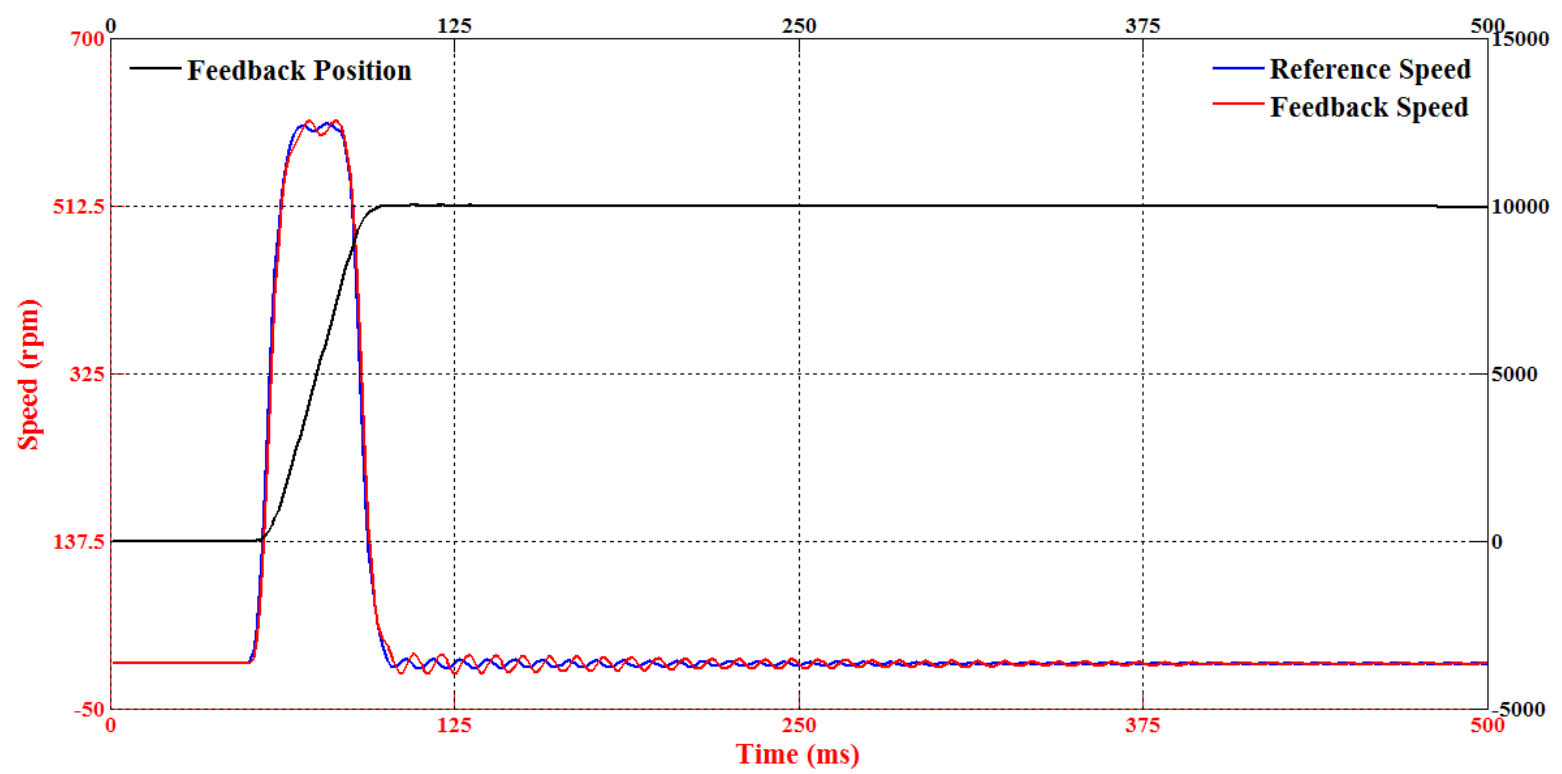

Figure 11. Experimental speed profile without input shaper

control performance.

\section{CONCLUSIONS}

The three input shaping algorithms for a flexible beam have been presented. A mathematical model of beam has been used to analyze natural frequency and damping ratio. According 


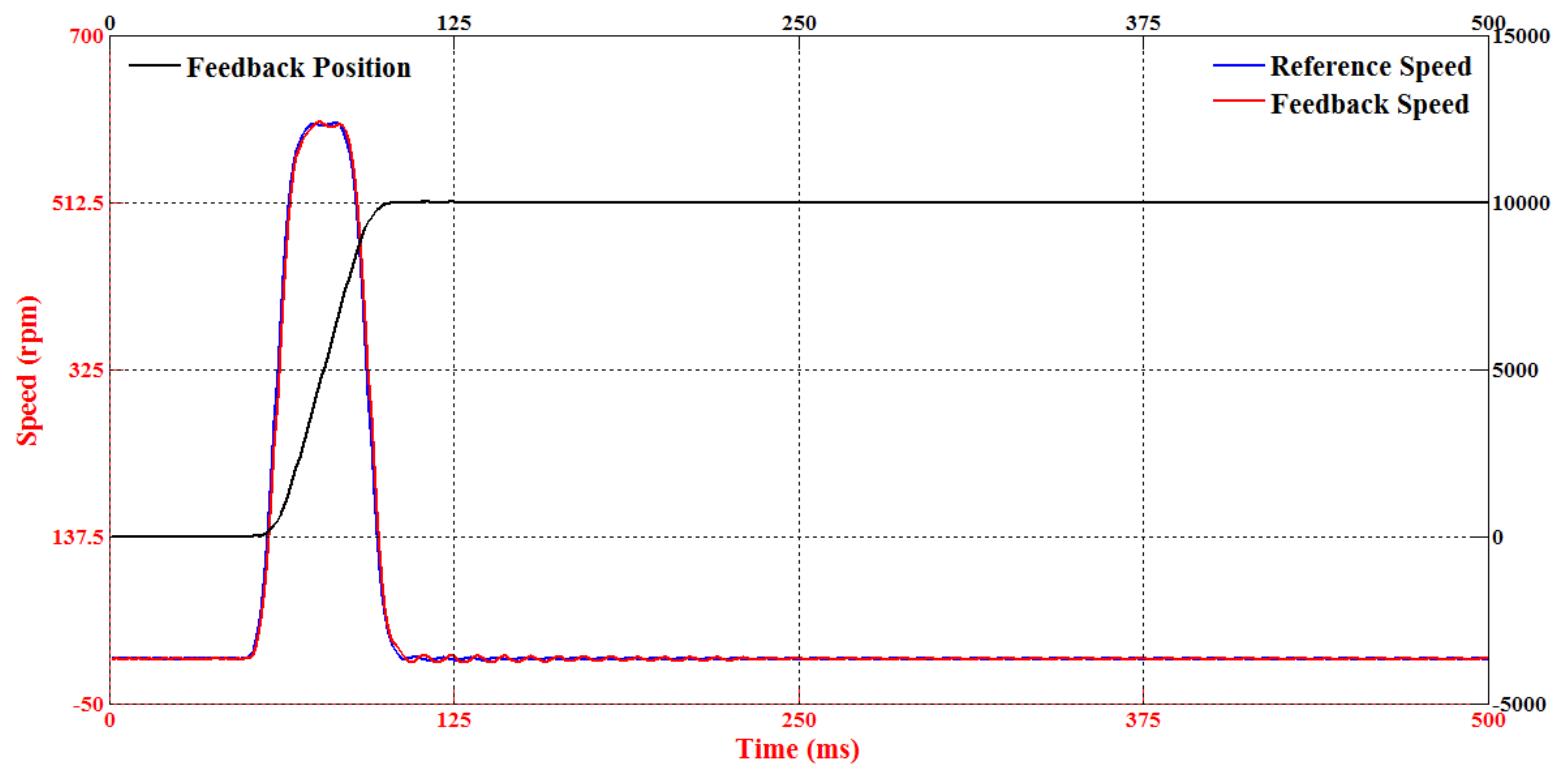

Figure 12. Experimental speed profile with ZV shaper

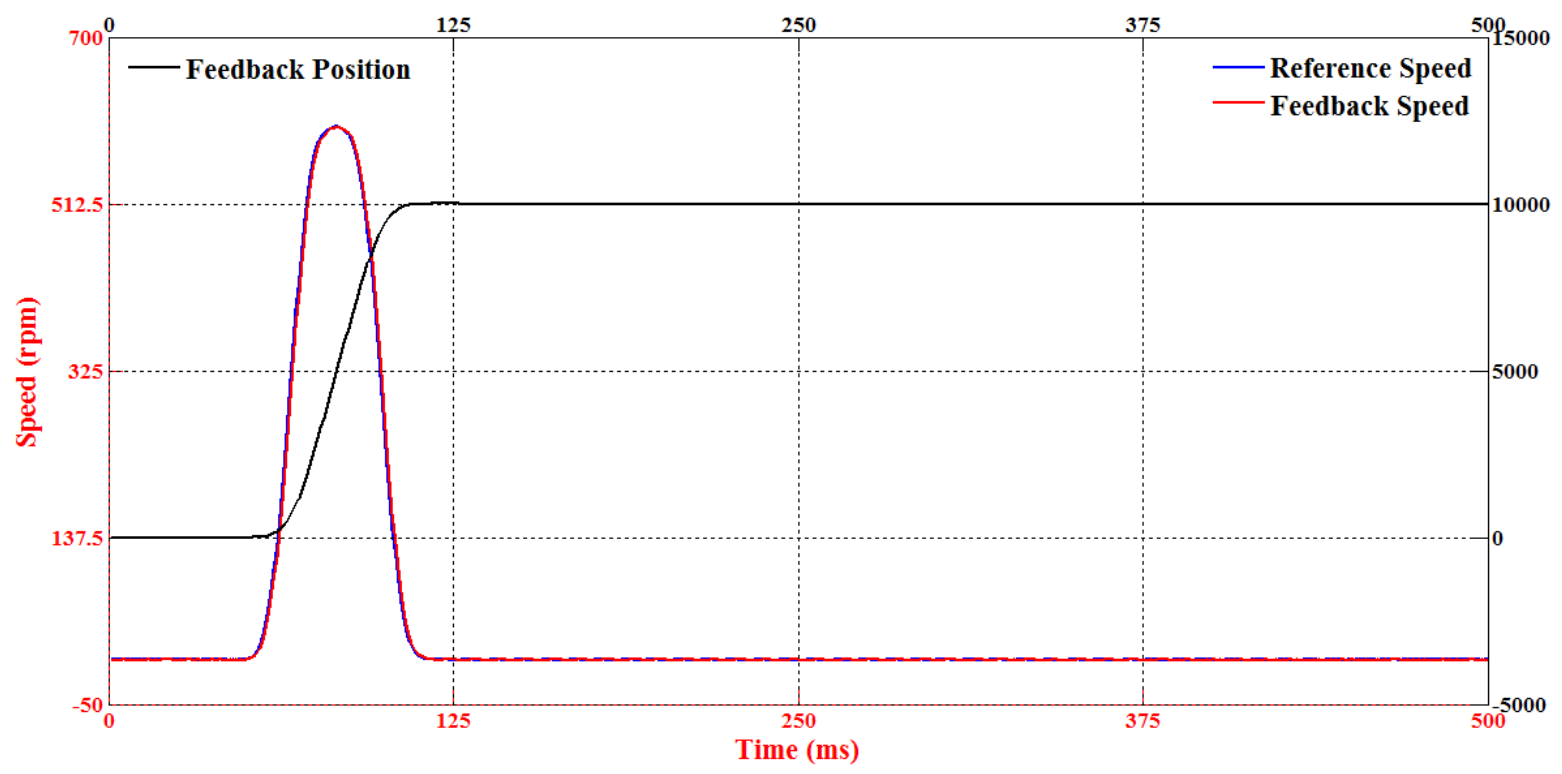

Figure 13. Experimental speed profile with ZVD shaper

to the experimental results, the motion profiles are smooth and reduced residual vibration. The implementation of the three input shapers is performed by using the Mechatrolink-III network. It is indicated that the input shapers have provided good control performance. With large buffer enough in Mechatrolink-III, this successful implementation provides an opportunity to apply the input shaper for multi-axes in industrial network motion system. 


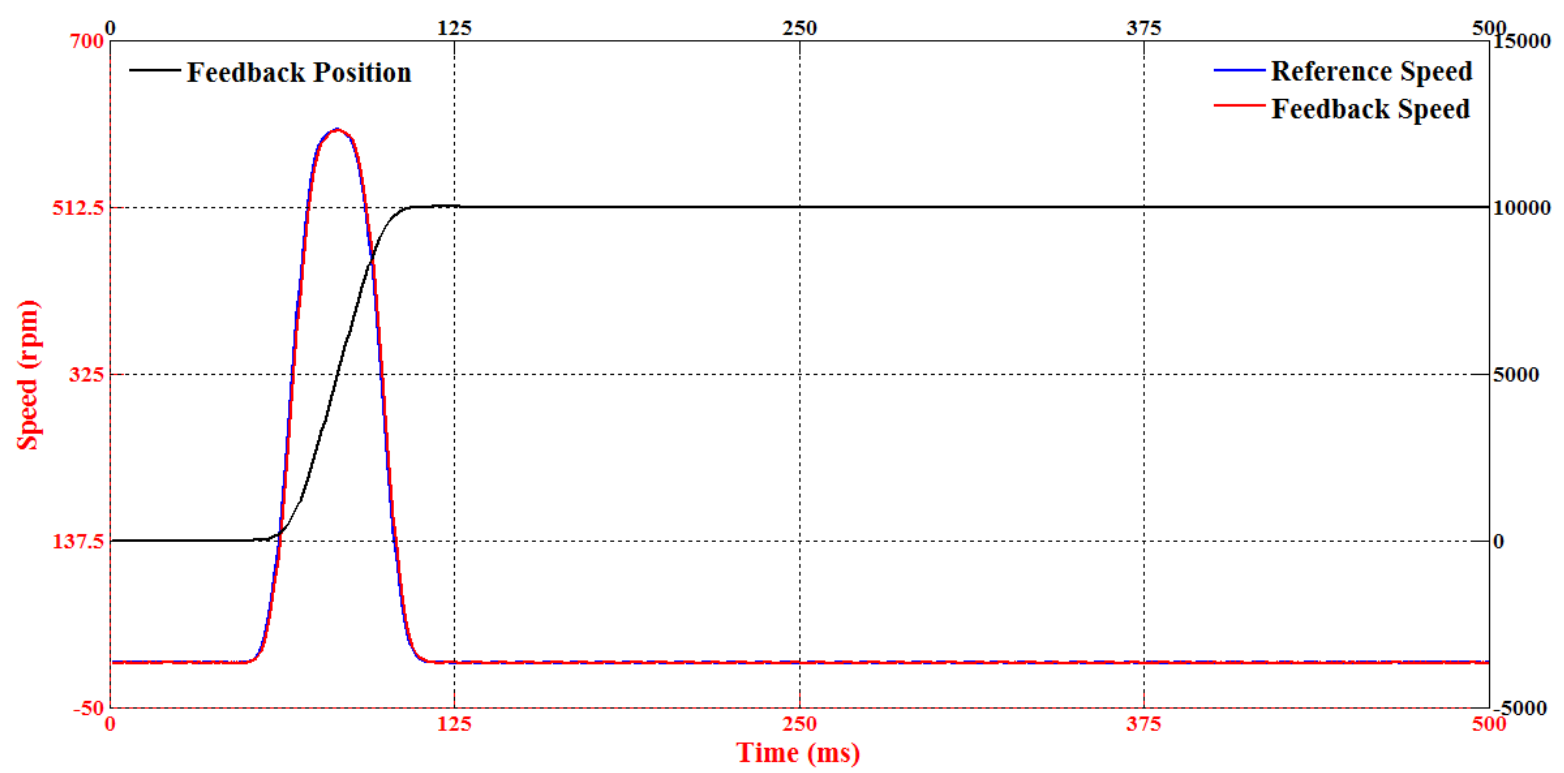

Figure 14. Experimental speed profile with ZVDD shaper

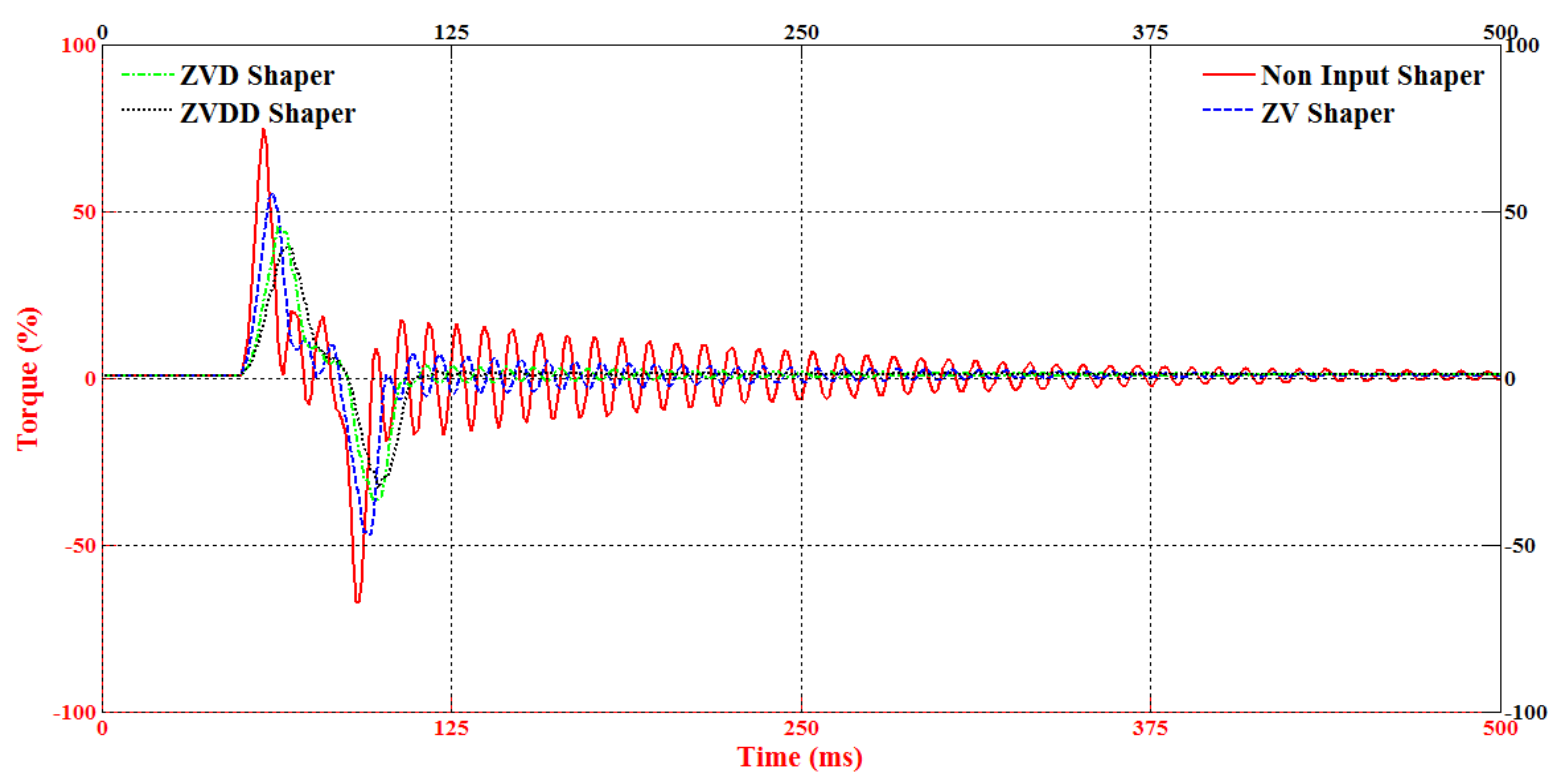

Figure 15. Experimental torque control with ZV, ZVD shaper, ZVDD shaper and without shaper

\section{ACKNOWLEDGMENTS}

This research is funded by Vietnam National Foundation for Science and Technology Development (NAFOSTED) under grant number 107.04-2012.37 and by Vietnam National University Hochiminh City (VNU-HCM) under grant number C2013-20-01. 


\section{REFERENCES}

[1] D.-M. Tsay and C.-F. Lin, "Asymmetrical inputs for minimizing residual response," in IEEE International Conference on Mechatronics, 2005. ICM'05. IEEE, 2005, pp. 235-240.

[2] F. Zou, D. Qu, and F. Xu, "Asymmetric s-curve trajectory planning for robot point-to-point motion," in Proceedings of the 2009 international conference on Robotics and biomimetics. IEEE Press, 2009, pp. 2172-2176.

[3] C.-W. Ha, K.-H. Rew, and K.-S. Kim, "A complete solution to asymmetric s-curve motion profile: Theory \& experiments," in Control, Automation and Systems, 2008. ICCAS 2008. International Conference on. IEEE, 2008, pp. 2845-2849.

[4] H. Li, M. Le, Z. Gong, and W. Lin, "Motion profile design to reduce residual vibration of high-speed positioning stages," IEEE/ASME Transactions on Mechatronics, vol. 14, no. 2, pp. 264-269, 2009.

[5] H. Karagülle, L. Malgaca, M. Dirilmiş, M. Akdağ, and Ş. Yavuz, "Vibration control of a two-link flexible manipulator," Journal of Vibration and Control, p. 1077546315607694, 2015.

[6] Ş. Yavuz, L. Malgaca, and H. Karagülle, "Vibration control of a single-link flexible composite manipulator," Composite Structures, vol. 140, pp. 684-691, 2016.

[7] W. Singhose, W. Seering, and N. Singer, "Residual vibration reduction using vector diagrams to generate shaped inputs," Journal of Mechanical Design, vol. 116, no. 2, pp. 654-659, 1994.

[8] M. A. Ahmad, A. N. K. Nasir, R. R. Ismail, and M. S. Ramli, "Comparison of hybrid control schemes for vibration suppression of flexible robot manipulator," in Computer Modeling and Simulation, 2009. ICCMS'09. International Conference on. IEEE, 2009, pp. 356-360.

[9] S. Gürleyük and Ş. Cinal, "Robust three-impulse sequence input shaper design," Journal of Vibration and Control, vol. 13, no. 12, pp. 1807-1818, 2007.

[10] V. P. Phan, N. S. Goo, K.-J. Yoon, and D.-S. Hwang, "Lookup table control method for vibration suppression of a flexible manipulator with optimization of the minimum settling time and energy consumption," Asian Journal of Control, vol. 14, no. 3, pp. 693-706, 2012.

[11] M.-T. Ha and C.-G. Kang, "Experimental analysis of natural frequency error to residual vibration in zv, zvd, and zvdd shapers," in Ubiquitous Robots and Ambient Intelligence (URAI), 2013 10th International Conference on. IEEE, 2013, pp. 195-199.

[12] "http://www.yaskawa.com."

[13] T. T. Dang, J. H. Kim, and J. W. Jeon, "Performance analysis of mechatrolink-iii," in 2013 11th IEEE International Conference on Industrial Informatics (INDIN). IEEE, 2013, pp. 152-157.

[14] X. Xu, F. He, Y. Tao, Z. Wang, and J. Xing, "Design and implementation of mechatrolink-iii bus slave station based on sopc," in Computational and Information Sciences (ICCIS), 2010 International Conference on. IEEE, 2010, pp. 1013-1016.

[15] N. C. Singer and W. P. Seering, "Preshaping command inputs to reduce system vibration," Journal of Dynamic Systems, Measurement, and Control, vol. 112, no. 1, pp. 76-82, 1990.

Received on August 24 - 2015

Revised on May 13 - 2016 